\title{
SUPPORTING AGILITY IN VIRTUAL ENTERPRISES
}

\author{
L. M. Camarinha-Matos ${ }^{1}$, H. Afsarmanesh ${ }^{2}$, R. Rabelo ${ }^{3}$ \\ ${ }^{1}$ New University of Lisbon, PORTUGAL \\ Quinta da Torre, 2825 Monte Caparica,Portugal, cam@uninova.pt \\ ${ }^{2}$ University of Amsterdam, THE NETHERLANDS \\ Kruislaan 403, 1098 SJ Amsterdam, The Netherlands, hamideh@wins.uva.nl \\ ${ }^{3}$ Federal University of Santa Catarina, BRAZIL \\ Campus Universitário, Florianopolis, SC, Brazil, rabelo@das.ufsc.br
}

\begin{abstract}
Agility is an important requirement for successful organizations in periods of market turbulence and unpredictable socio-economic changes. Agility in a virtual enterprise depends on the skills of its member enterprises and the characteristics of its supporting infrastructure in this paper. Various requirements and approaches for agility support during the VE life cycle are presented and topics requiring further research are identified.
\end{abstract}

\section{INTRODUCTION}

Recent trends in industry are emphasizing the relevance of agility, understood as the ability to recognize and rapidly react and cope with the unpredictable changes in the environment [Goranson, 1999], [Kidd, 1994].

Being defined as a temporary consortium of enterprises that join skills and resources, supported by computer networks, to better respond to a business opportunity, the concept of virtual enterprise (VE) embeds an implicit notion of agility. It is commonly associated to this concept the idea of highly dynamic organizations that form themselves according to the needs and opportunities of the market and that remain operational as long as these opportunities persist. Once the business case ends, the organization dismantles itself although its members will continue to exist and will probably participate in other such dynamic consortia. The composition of a VE is determined by the need to associate the most suitable set of skills and resources contributed by a number of distinct individual organizations.

In this context the VE suggests, in principle, an inherently agile organization. This does not necessarily mean that each member of the VE is an agile enterprise. In fact there is even the risk that companies that are overspecialized in their core competencies, and especially in the case of SMEs with limited resources, become vulnerable when the changes in the market determine that their core skills are less important. Several authors have advocated outsourcing and the need for companies to focus on a reduced set of core competencies, towards lean enterprises, in order to reach world class in those areas. In fact, this is already happening at some enterprises over the world, which put more emphasis on production optimization than on product flexibility. From a more abstract point of view, the competitiveness

The original version of this chapter was revised: The copyright line was incorrect. This has been corrected. The Erratum to this chapter is available at DOI: 10.1007/978-0-387-35399-9_52 
required from the enterprises in a global market is slightly changing some earlier "academic forecasts" that pointed out the need for extreme high degree of flexibility in industries. It is right that the large enterprises, typically the core industries in an extended/virtual enterprise or in a supply chain, keep a level of flexibility in all levels. However, the "satellite" enterprises are becoming more optimized, producing a lower product variety.

The highly unpredictable directions of market change and the fast speed of this change suggests however, that in order to survive companies should keep a balanced set of competencies and the capability to develop new competencies even at the price of being less lean.

On the other hand, the actual presence of agility in a VE depends on a number of technical, legal, cultural, and socio-organizational factors. In technological terms there is a need for a highly flexible, secure and robust infrastructure to support agility along the life cycle of the virtual enterprise. The legal framework of the countries may represent an obstacle to agility, especially when there is not yet a wide body of knowledge on cooperation agreements and contractual regulations for this type of organizations. As pointed out by Goranson, companies operating under code-based laws, requiring extensive contract definitions, show less agility than those operating under a case-based law context [Goranson, 1999]. Additional difficulties arise when a VE is composed of enterprises operating in different legal frameworks. Finally the cultural and socio-organizational aspects are key issues for both the implantation and agility of the VE paradigm. Creating a culture of cooperation, trust building, bridging inter-cultural differences, redefining the internal organization of the companies, defining new internal roles, and planning appropriate training programs, are major challenges faced by enterprises.

This paper is mainly focused on the technological requirements to support agility in a VE. The discussion of these requirements and proposed solutions follows the typical major phases of the VE life cycle, namely the creation, operation, reconfiguration, and dissolution [Camarinha-Matos, Lima 1998][Spinosa et al., 1998]. This work is based on the research activities and experiences acquired in various European projects, with particular relevance to the Esprit PRODNET II [Camarinha-Matos and Afsarmanesh 1999] and INCO-DC MASSYVE [Rabelo et al. 1999].

\section{VE FORMATION}

\subsection{Major steps}

Once a business opportunity is detected, there is a need to rapidly plan a VE, identify partners, and establish cooperation agreements to regulate the operation of the VE. In order to help the VE creator (VE coordinator, VE architect, or broker) the support infrastructure should provide a set of specific service functions.

Some of the major steps and required functions in VE creation were identified by PRODNET (Fig. 1). The first two steps of setting up infrastructure resources and manifestation are preparatory operations to make a company ready, from the infrastructure point of view, in order to participate in VEs which end with the registration of the company in a public directory or cluster of enterprises willing to cooperate. When a business opportunity is detected, there is the need to plan and 
create the VE, establish the contract or cooperation agreement among partners, and configure the infrastructure to operate according to this agreement.

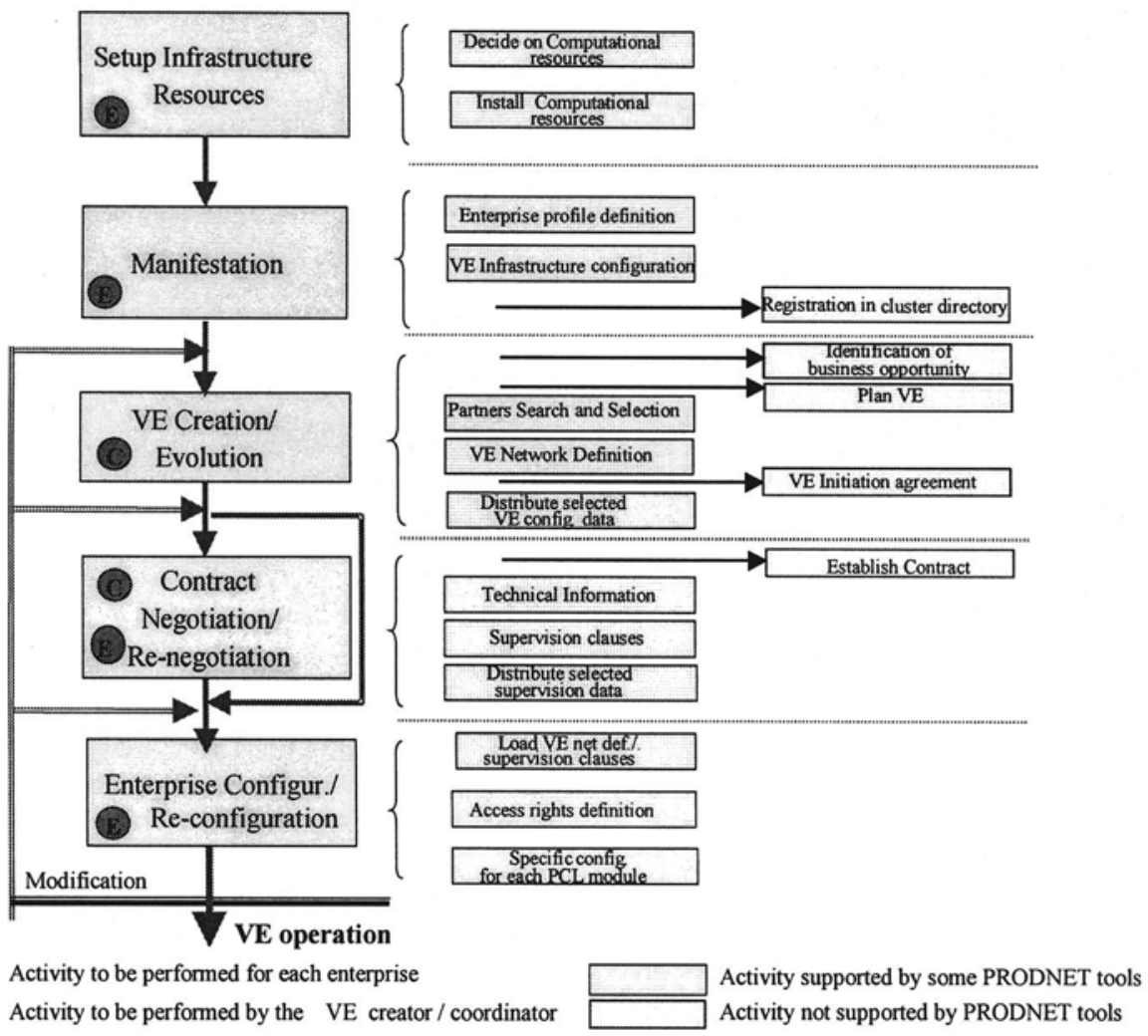

Figure 1 - Major steps in VE creation

PRODNET II developed some tools to support some parts of the creation process. Other projects such as the Brazilian VIRTEC [Bremer et al., 1999], the ALFA COSME-VE [Molina et al., 1998], and COWORK [Alzaga and Martin, 1999] have addressed the specific aspects of creation and management of clusters of enterprises that are willing to cooperate as well as the planning of the VE structure for a given business opportunity. Further work is necessary in order to develop planning tools integrating the VE planning, the high-level business processes modeling, the resources planning and the capacity planning.

\subsection{Partners search and selection}

The selection of business partners is a very important and critical activity in the operation of a company. Similarly, and even in a more acute way, selection of partners is a very important process in the life cycle of a VE.

Partners search can be based on a number of different information sources, being private, public, or independent. The enterprise's private suppliers' list - an important asset of a company - is a data repository that contains information about the 
companies that have had commercial relationships with this enterprise. This information is usually maintained by the ERP or PPC System and composes an Internal Suppliers Directory (ISD). External sources include directories maintained by industrial associations, commerce chambers, or Internet services already available for several countries and that offers WWW interfaces to a database of enterprises served by a local search engine, the External Suppliers Directory (ESD). Another emerging solution is the creation of clusters of enterprises that agreed to cooperate and whose skills and available resources are registered in a common Cluster Directory (CD).

The search based on Internal Suppliers Directory can take advantage of previous knowledge of partners and cooperation experience, leading to higher confidence. The suppliers are usually ranked according to some particular criteria and specialized decision support systems can be used to speed up the partner's selection process. But even when this information source is available, for some business opportunities it might be necessary to look for new partners when new skills or more resources are necessary. In PRODNET II both Internal and External directories were considered. Fig. 2 illustrates the main steps when searching for partners in Internet based directories [Camarinha-Matos and Cardoso, 1999].

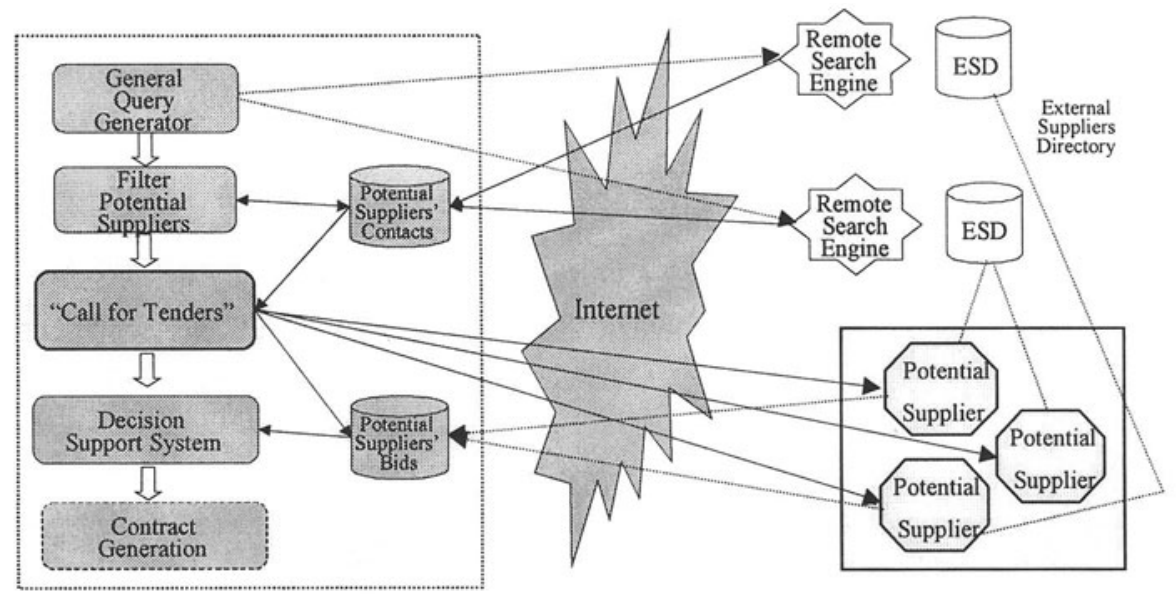

Figure 2 - Partners search and selection based on external directories

A typical difficulty - and an obstacle to agility - is the dependence on the specific formats and access interfaces of each ESD. The lack of a standard interface and standard representation of enterprise profiles does not facilitate the development of generic tools.

These problems are eliminated when the search is restricted to controlled clusters of partners. Within a cluster it is possible to establish a common model for the enterprise profiles tailored to the needs of a broker responsible for VE creation. The VIRTEC, COSME-VE, and COWORK projects are examples of this approach. Similarly to the case of Internal Suppliers Directory, the Cluster Directory can maintain knowledge about the past cooperation experiences and partners performance, constituting important information for the partner search phase. The 
cluster directory, although maintained by a cluster manager, is available to the various members of the cluster that can take the initiative to create a VE.

One approach to partners search within a cluster is proposed by the MASSYVE project. Instead of resorting to a centralized directory, a multi-agent approach based on the negotiation paradigm is adopted. The proposed system includes a broker agent responsible for handling business opportunities, a facilitator, consortium agent(s) that design and plan the potential $\mathrm{VE}(\mathrm{s})$ and negotiates with potential partners within the cluster, and a set of agents representing the enterprises participating in the cluster. Once a business opportunity is acquired, the facilitator makes a pre-evaluation of possible partners by comparing the required skills and resources with the profile and current status of the cluster members. The contract-net protocol [Smith, 1980][Davis and Smith, 1983] is then used to collect bids from cluster members and select the most adequate ones based on the costs and delivery dates for instance. Figure 3 illustrates this process. In case the cluster does not cover all the requirements, a more general search for partners can be performed through Internet directories of enterprises.
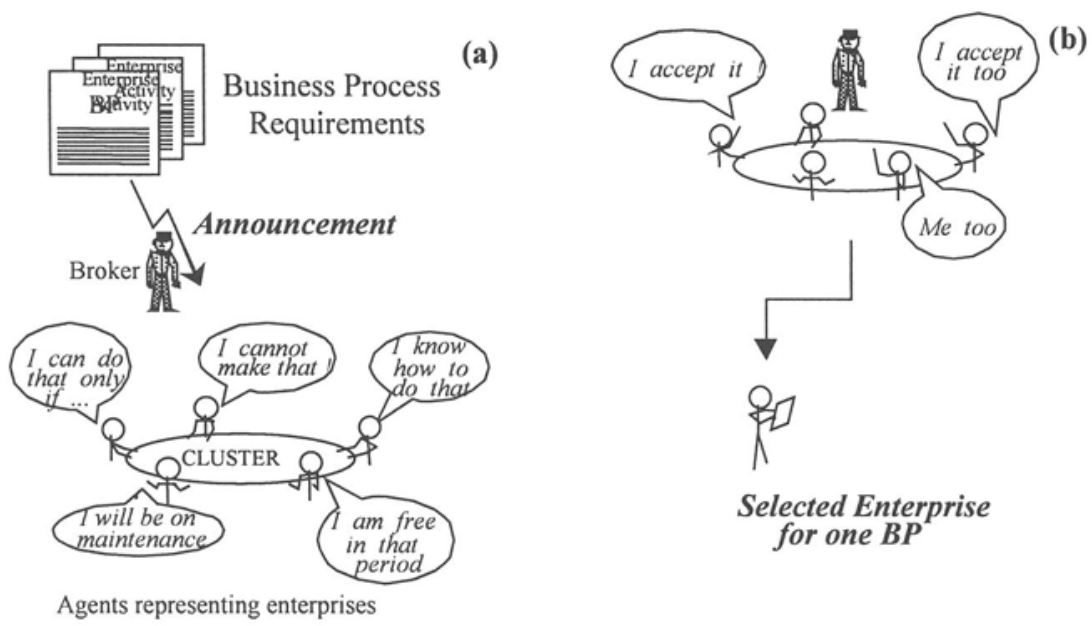

Selected Enterprise
for one BP

Figure 3 - Negotiation applied to a cluster

Another approach to implement a flexible partners search is based on the concept of federation of services as for instance proposed by JINI [SUN, 1999]. The JINI architecture can support, in a transparent way, federation of service functions offered by different service suppliers and running on different nodes of a network. Special directories -JINI Lookup Services- provide the registration of service specifications and the associated functions to search such services based on a configurable search criterion, see Fig. 4. According to this model, enterprises should be able to plug/unplug their services to/from the service directories, and clusters must provide the proper "standard" service interface. Service interfaces aim at supporting the interoperability with other (requesting) enterprises, regardless of the heterogeneity associated with the actual implementation of the services themselves. This means that no matter how the service is actually implemented, in terms of the computer platform, operating system, programming language, internal modules, etc., there is a 
client service interface that can be managed by the lookup service and made available to other enterprises that may request it.

Although JINI may contribute to facilitate the interoperability among services offered by different enterprises, its use in VE environments requires the development of specific additional functionalities. For instance, in VE the matter of access rights and enterprises information visibility is a very important issue and therefore when searching and accessing a specific service type it is also relevant to determine both the supplier of the service and the requesting client, in order to check such visibility rights. It is also necessary to define the rules for service specification and registration through the service interface. Furthermore, the general acceptance of the service interface by the service providers for developing services compliant with such rules is of great importance. This can be easier achieved within the context of a cluster of enterprises. Therefore the JINI system for instance can be used as an extension to the current cluster directories of enterprise profiles.

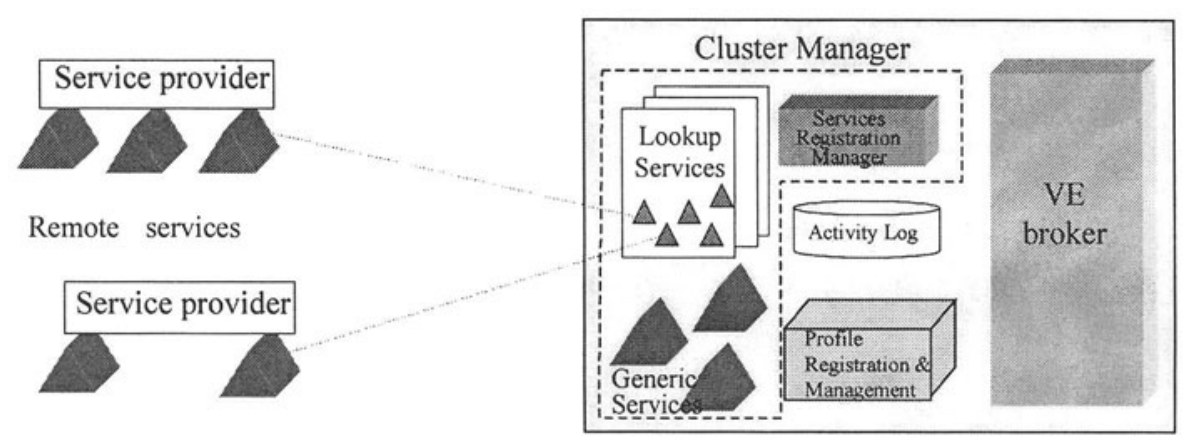

Figure 4 - Jini-based cluster manager

\subsection{Configuration aspects}

The ease of configuration of the VE, using the VE support infrastructure, is a major requirement for agility. In order to cope with the diversity of virtual organization types and different business practices applied to different companies, the VE support infrastructure should allow an easy configuration of desired behavior.

Configurability was a major design requirement in the PRODNET infrastructure. PRODNET provides tools for configuration of its different services (i.e. besides the internal configuration of each of the infrastructure modules). For instance, to support enterprise's autonomy in definition of its behavior and the rights for other enterprises, PRODNET provides:

- Workflow-based graphical definition of the VE nodes behavior regarding each cooperative event, via the node's local activity coordination module.

- Configuration of data access rights and information visibility levels, via the export schemas in the node's federated information management module. These schemas are defined at the VE-members providing the proper visibility rights for the VE coordinator according to the supervision clauses defined in the VE contracts. 
- Setting and configuration of the used common technology for the VE, and the communication characteristics, via the selection of data exchange standards (EDIFACT, STEP, ...) and security mechanisms (cryptography, digital signature, ....).

In spite of these tools, a VE infrastructure as proposed by PRODNET, involves many technologies and paradigms, each one requiring specialized knowledge for installation and configuration. Although a more automated installation is desired, this requires a more stabilized technology, as well as special consideration at the system design phase. In either case, the configuration effort is always larger for the first time that an enterprise participates in a VE, since at this stage also the socioorganizational issues and all the required business processes reengineering need to be addressed to properly support the enterprise for its VE participation.

\section{VE OPERATION}

Agility is also required at various levels during the VE operation, in order to cope with the unexpected changes in the environment.

\subsection{Agility and distributed business process coordination}

When a Business Process (BP) is executed by a virtual enterprise parts of the decomposition of this BP (i.e. sub-processes) are assigned to different enterprises, becoming a Distributed Business Process (DBP) [Rabelo et al., 1996] or a virtual business process in the WISE terminology [Alonso et al., 1999]. The problem of the supervision or coordination of a DBP at its various levels of decomposition becomes even more important in this context where its definition and enactment is not limited to a single organization, but instead to a set of autonomous, distributed and heterogeneous nodes need to cooperate. When properly "orchestrated", the various processes taking place in the different members of the VE are expected to lead to the achievement of the global goals of the VE.

The VE coordinator is responsible for the entire DBP execution, while the VE members are responsible for the coordination of the sub-BPs assigned to them. The VE coordinator may itself act also as a VE member. As such, the VE coordinator can also be responsible for a particular sub-BP. A VE member may itself become the coordinator of a sub-VE inside the VE to coordinate (or supervise) its sub-BP. Under this approach, temporary (sub-) consortia can be formed inside a VE and so on. These sub-consortia are formed for the sole purpose of facilitating the coordination of activities involved in the related sub-business processes. Once a subbusiness process ends, the sub-consortium "dissolves" and its members may become involved in other sub-consortia dynamically formed in this or other VEs as the execution of the VE BPs evolves. For instance, the enterprise C (in Fig. 5) coordinates sub_VE1 and is a member of sub_VE2.

Once a global business process is defined, scheduled, and responsibilities are assigned to each individual partner, the successful achievement of the common goal - delivery of the final product to the client - depends on the proper and timely operation of each VE member (and each supporting service in each VE member). A delay in one node, if not properly attended in time, may jeopardize the common VE 
goal. Therefore, it is necessary to manage (supervise) the inter-dependencies among the various (distributed) BPs.

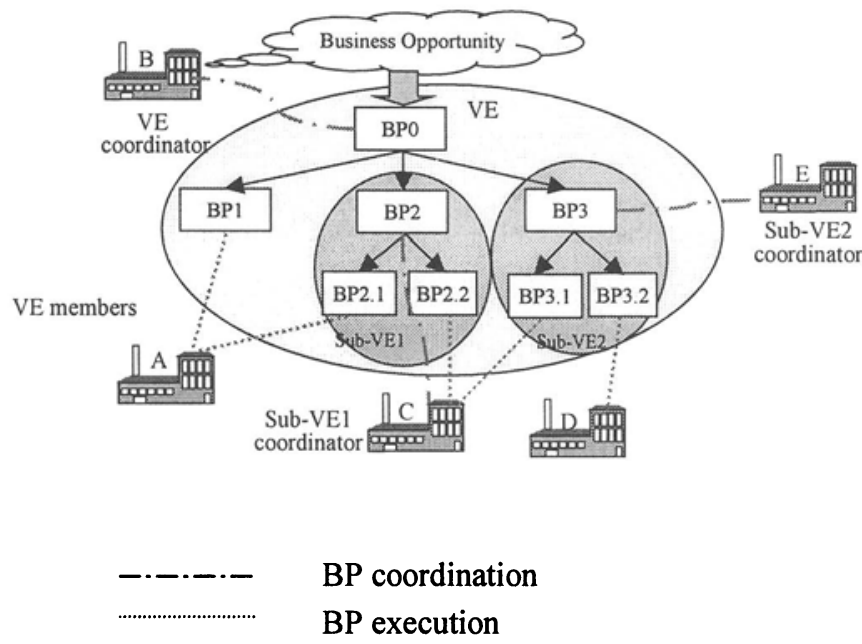

Figure 5 - Example of DPB execution

Several languages and formalisms have been used for BP modeling and coordination. In the VE area many projects, such as the case of PRODNET II [Camarinha-Matos and Lima, 1998], adopted a workflow-based approach due to both availability of experience with workflow systems in many enterprises and the standardization efforts promoted by the Workflow Management Coalition [WfMC, 1994].

Two major but distinct approaches have been followed by different research projects focused on Virtual Enterprises. While flexible infrastructures are not properly addressed, a few projects have followed a bottom up approach trying to support the main functional VE requirements for both the information exchange and basic coordination of inter-enterprises activities. Once these services are guaranteed, the efforts are progressively directed towards the support of the BP execution. Meanwhile, a second group of projects have devoted their efforts to the identification of the major BPs and their decomposition in some key areas of manufacturing, without paying enough attention to the base VE infrastructure requirements. However, although important, modeling BPs bring little benefit to the actual operation of the VE, if the VE is not supported by an execution infrastructure - namely the $\boldsymbol{V E}$ execution system. It is therefore necessary to "close the gap" between these two approaches.

PRODNET II, although mainly aims at the first approach, also addresses the coordination of BPs at different levels of abstraction, see Figure $6 \mathrm{a}$.

The proposed infrastructure extends the functionalities of each VE member (represented by the enterprise applications such as ERP/PPC, PDM, CAD, etc.) with a Cooperation Layer responsible to handle all enterprises cooperation events. Central to this coordination kernel are a Distributed/Federated Information Management System and a workflow-based Coordination Engine. A safe 
Communications Infrastructure and a library of support services complete the Coordination Layer; see Figure $6 \mathrm{~b}$.

The complete description of the layered hierarchical coordination architecture of PRODNET is described in [Camarinha-Matos and Lima, 1999].

In fact, in addition to the VE coordination role, responsible for the global BP, other enterprises may assume the role of coordinators of sub-business processes that might be decomposed and performed by a sub-consortium of enterprises. This idea of dynamic formation of sub-consortia inside a community of cooperating agents (a $\mathrm{VE}$ ) in order to coordinate sub-business processes is being exploited by the MASSYVE project [Rabelo et al., 1999], providing higher levels of agility within the enterprise. The process is similar to the one illustrated in Fig. 3 but the set of target agents is now restricted to the members of the VE and not the full list in the cluster.

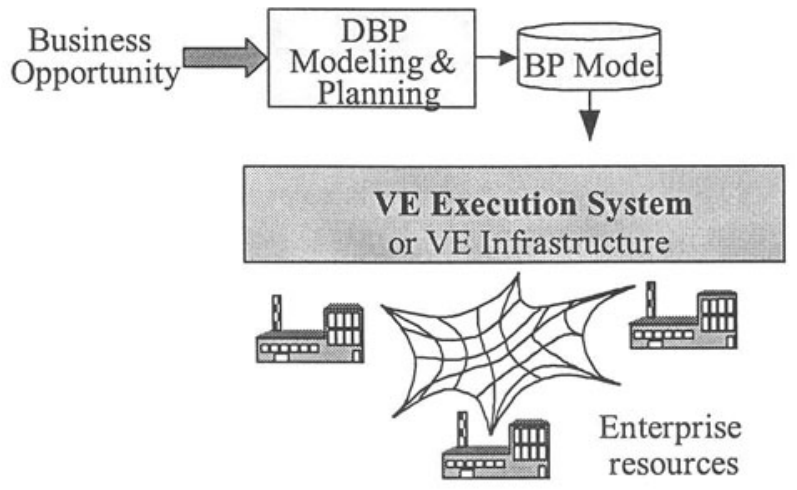

Figure 6.a - VE execution system

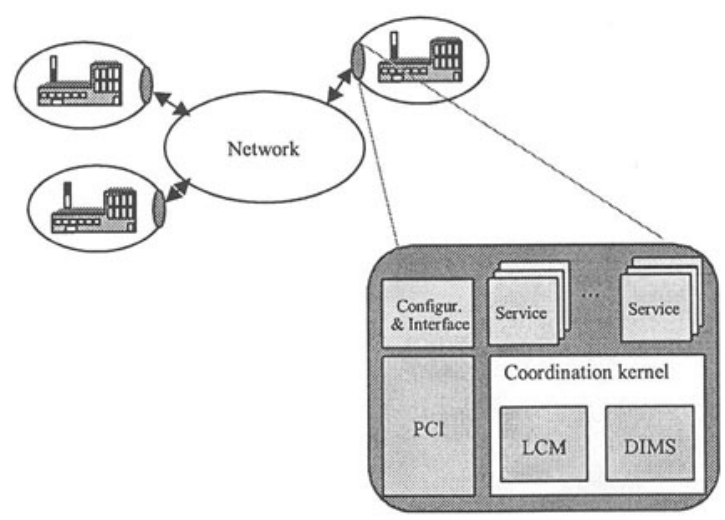

Figure 6.b- PRODNET approach to VE execution environment

\subsection{Support full or controlled transparency to the customer}

Interaction with the customer during the full life cycle of the business process is a major facet of agility. New management objectives focused on pursuing customer 
satisfaction imply not only the supply of up to date information to the customer, but even his/her involvement in the process.

Regarding the information access, once an infrastructure such as the PRODNET Cooperation Layer is established, adequate information visibility / access levels for the customer can be easily defined using the mechanisms provided by DIMS and DBPMS modules and a set of specific monitoring workflows. But true agility is achieved when the customer is not just a passive observer but also an actor having some capability to intervene in the process. For instance, in some cases, the customer may change the order requirements or be an active member in a conflict resolution (re-scheduling, for instance) process. For this purpose a new type of actor - the customer - has to be defined in the VE environment and specific interface front ends provided by the VE coordinator (via a web browser, for instance).

It shall be noted that some logistic operators already provide some primitive steps in this direction that in addition to giving the customer some information about the status of his/her order, also allows them some interaction (even cancellation in some cases). A similar approach can be extended to the manufacturing process as well. One approach in this direction is supported by the PRODNET DBPMS module, which by E-mail periodically sends "production bulletins" to the customer, both in text and html formats.

Furthermore, to better support customer's monitoring of the activities related to the order, a "push mechanism" needs to be implemented. In PRODNET three modules can support the implementation of this push mechanism. On one hand, the configuration of information visibility levels, supported by the federated information management of the DIMS module, and on the other hand the configuration of the contract-based monitoring functionalities at the DBPMS module, can together, with the support of the Local Coordination Module, provide the base platform for pushing the status information from the VE-members to the coordinator, that in turn can make it available to the customer.

Another perspective is related to a wider vision of the inventories and enterprises capacities aiming at a better supply chain management. The scope of SCM under an integrated logistics management covers the flow of goods from supplier through manufacturing and distribution chains to the end-user. This concept suggests a holistic approach to material and information flow management. Partnership and trust aspects are introduced as important elements in this kind of supplier relations. Actually, the basic notion of supply chain management is grounded on the belief that efficiency can be improved by sharing information and by joint planning. Therefore, a global and more profitable supply chain can be achieved in the sense of more balanced / negotiated planning based on an entire optimum perspective [Klen et al., 1998].

\section{VE RECONFIGURATION}

During the operation of a VE it might be necessary to change some partners or change the roles of some partners. This is the case, for instance, when there is a scheduling conflict to resolve at the DBP level. A partial solution is given by the DBPMS module of PRODNET [Klen et al. 1999]. A more general solution can be achieved with dynamic scheduling solutions as proposed by the MASSYVE project. 
MASSYVE is investigating the application of multi-agent approaches to agile scheduling in virtual enterprises. A component-based approach is adopted in this project that integrates previous work on multi-agent based scheduling inside an enterprise (the HOLOS system [Rabelo and Camarinha-Matos, 1996]) with the federated information management (the DIMS/FIMS system [Afsarmanesh et al., 1999] [Garita et al. 2000]) and extends the approach to VE [Rabelo et al., 1999][Rabelo et al., 2000].

A multi-agent scheduling system is composed of a set of "processors" (nodes in a network of manufacturing resources), each one with its own particular capabilities (typically heterogeneous), that have to exchange and process information in order to contribute to finding a solution to the global scheduling problem. These agent nodes constitute either enterprises when the scheduling problem is discussed at the VE level, or the internal manufacturing resources of a company when dealing with the internal scheduling of tasks assigned within a company.

Several problems can arise during the schedule generation, after its generation, and during its execution, such as the temporal, capacity, or technologic conflicts. These problems come from the planning, scheduling or execution supervision activities. There are several methods that can be applied for conflict resolution in a multi-agent system. MASSYVE uses the Contract-Net Protocol [Davis, 1980] coordination mechanism to support the task assignment among the agents, and the Negotiation [Davis and Smith, 1983] method to overcome conflicts taking place during one of the three mentioned scheduling phases.

The proposed agile scheduling system is a collection of classes of agents configured for a particular scenario. For each of the two levels - VE or enterprise level - four classes of agents (fig. 7) described below are responsible to perform certain coordination tasks:

- Scheduling Supervisor (SS): is the class whose (unique) instance performs the global scheduling supervision.

- Enterprise Activity Agent $(\boldsymbol{E A A})$ : is the class whose instances are associated to the production resources, i.e., resources are represented by EAA. These agents are the executors of tasks.

- Local Distribution Center (LDC): is the class whose instances represent functional clusters of EAAs in order to avoid announcement broadcasting and hence to make the selection of an EAA faster. They are also responsible to select the most suitable agent for a certain task after the negotiation process.

- Consortium $(\boldsymbol{C})$ : is the class whose instances are dynamically created to supervise (locally and in a decentralized way) the schedule of a logical arrangement of EAAs selected to execute an entire task or BP.

When the scenario is intra-organizational scheduling (i.e. scheduling of the tasks / BPs assigned to a particular enterprise), these classes can have the following interpretation:

- The Scheduling Supervisor is the coordinator for all the tasks assigned to the enterprise.

- The Enterprise Activity Agents represent the various internal resources. For instance, an EAA can be associated to each machine in the shop floor, being responsible to acquire new contracts to this machine and to report about its status. 
- A Local Spreading Center represents a cluster of internal resources.

- A Consortium is a temporary association of agents involved in the execution of a tasks or BP inside the enterprise. Please note that a given BP awarded to a company by a VE supervisor can be further decomposed (internally) in a subtree of BPs and enterprise activities.

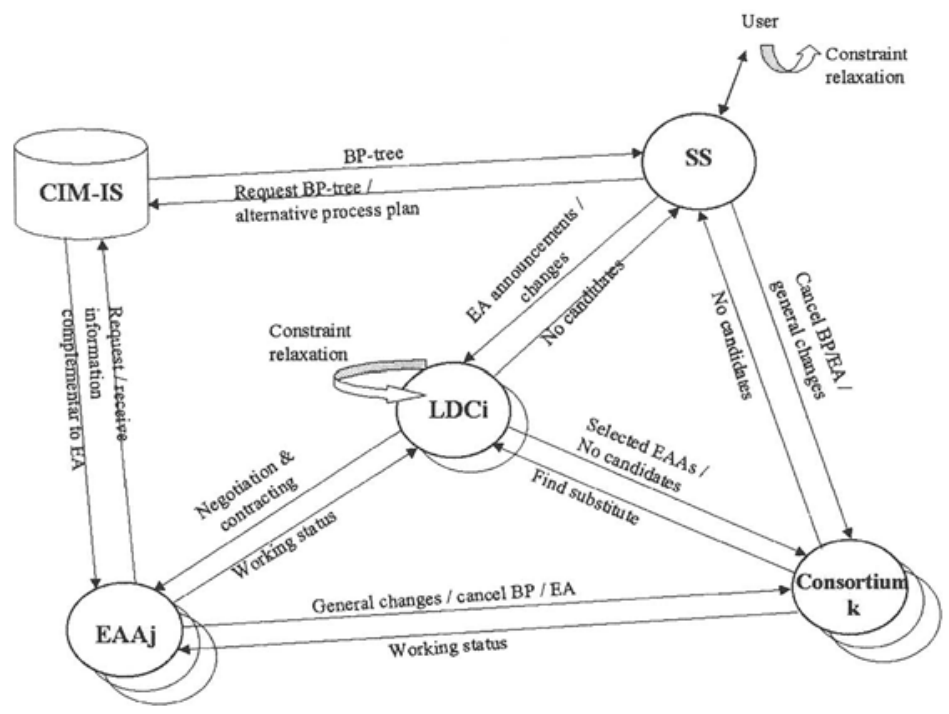

Figure 7 - Classes of agents in an intra-organizational scheduling scenario [Rabelo et al, 1996]

For the inter-organizational scheduling (i.e. high level scheduling of BPs at the VE level), the following interpretation applies:

- The Scheduling Supervisor is the global coordinator of the DBP, running at the VE coordinator node.

- The Enterprise Activity Agents are representatives of the VE members. Therefore, an EAA representing an enterprise coincides with the internal SS of that enterprise.

- The Consortium corresponds to a group of agents, representing a subset of enterprises that are involved in the execution of a given BP.

Figure 8 illustrates this scenario. There are two enterprises shown in this picture, $\mathrm{A}$ and $\mathrm{B}$. Since $\mathrm{A}$ has triggered the entire process of contracting and managing the production of a given product, it is viewed as the business process coordinator. Through the Internet, A can manage the suppliers (B, in this case) involved in this production/business. Each of the involved enterprises is represented by an agent the enterprise-agent - acting in the same perspective that a Scheduling Supervisor does. At this level, an enterprise-agent supervises a set of Consortium agents. At the Consortium level, each Consortium supervises a set of EAA agents. At the EAA level, each EAA agent supervises one (or more) machine(s).

Both intra-organizational and inter-organizational schedules are generated and supervised via a cooperative and tightly coordinated information exchange among agents. The first implementation of this system for a single enterprise, adopted a 
centralized information system (CIM-IS), see Figure 7. When a VE scenario is considered however, a more flexible information management approach is required and thus a federated system is adopted.

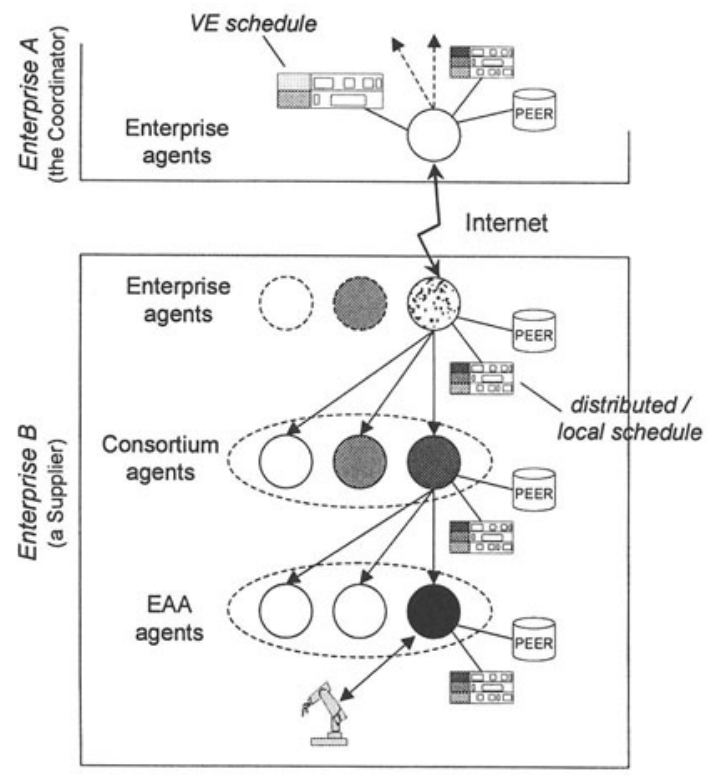

Figure 8 - Inter-organizational scheduling scenario.

In a MAS approach, the cooperation among agents is traditionally supported via message passing, with the communications protocol acting as the vehicle to transport control and data together. An interesting concept investigated in MASSYVE is that the data is not sent from one agent to the other via a high-level protocol, as in the traditional push strategy, but rather through the pull strategy via the access to a federated information management system (FIMS) associated to each agent [Rabelo et al., 1999]. Thus, the high-level protocol is used for coordination purposes only. The message's content then becomes shorter and leaner and the agents have access to the necessary data only when needed.

Figure 9 illustrates this approach. Consider the case that a given enterprise (B) processes some information and generates some results (for example $\mathrm{r}-17$ ) that should be sent to another enterprise (A). Then Agent-B sends a message to Agent-A (represented by 1), communicating that certain information ( $r-17)$ is available in its local database (that can be accessed through its export schema). Please notice that the access rights for the shared data among nodes is dynamically and bilaterally configured, according to the role and the needs of every node and preserved through the export schemas. Therefore, once this message is received at Agent- $\mathrm{A}$, whenever it wishes A can retrieve this updated information ( $\mathrm{r}-17)$ from $\mathrm{B}$. However, this access and transfer of information does not occur between the two agents, rather it occurs between the two information management modules (FIMSs) of the two enterprises. Namely, enterprise-A, through its integrated schema at the FIMS sends a request to access $\mathrm{r}-17$ to FIMS-B (represented by 2), using the federated mechanisms for information access - that receives the authorized information ( $\mathrm{r}-17)$ 
from B (represented by 3). The details of this interaction are outside the scope of this paper and the subject of forthcoming publications.

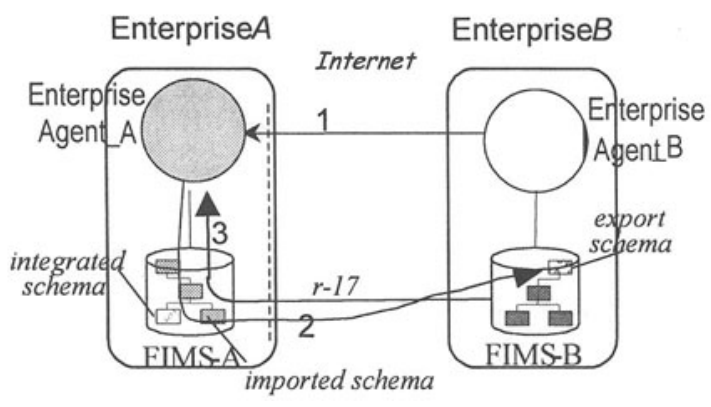

Figure 9 - Pull-based data exchange among the agents

Considering the extreme importance of trust building among the enterprises involved in a VE, preservation of the information visibility and access rights is a main concern of all autonomous enterprises. Therefore, the possibility of configuring, on a bilateral basis via the federated information management mechanisms [Afsarmanesh et al. 1999a] [Frenkel et al. 2000], the specific access rights to data owned by one agent for every other agent in the community, represents an important functionality. This functionality in turn supports the adequate levels of autonomy required by each enterprise in a VE environment, which constitutes another advantage regarding the traditional MAS approaches.

In the general case when there is a need to find partners outside the consortium in order to solve a conflict, the same tools used during VE formation can be used, but there is a need to define liabilities / future responsibilities and rights for "leaving partners".

\section{VE DISSOLUTION}

As stated in [Metes et al. 1998], "an organizational marriage is much easier to achieve than an organizational divorce". There are, in general, consequences of the operation of a VE that cannot be simply discarded when the VE dissolves. Most of these consequences are of a legal nature and shall be regulated by the cooperation agreements. That is the case, for instance, of the responsibility of customer support / product maintenance during the life cycle of the product / service generated by the VE. Environment regulations are also forcing companies to plan provisions regarding the product disposal and recycling after its end of life.

Recent regulations in some countries also state that the liabilities regarding each component of a product may ultimately lie with the component's supplier. In the case of a network chain type of manufacturing this forces each node in the chain to keep track of the history of each component /sub-product that "passed by" this node. This is a functionality that is properly supported by most of the more advanced ERP systems.

There are however other several less "material" issues that are more difficult to handle. One of these issues is the IPRs policy, namely for the post-dissolution phase and its consequences in terms of information accesses by the VE members. In some 
cases there is also the possibility that the $\mathrm{VE}$ evolves into a more permanent organization, a joint venture enterprise created by the VE members, to exploit the intellectual and industrial property results developed in cooperation.

From an information systems perspective it is also necessary to offer a set of configuration tools that allow each enterprise to cancel / limit the access rights of its former partners once the VE ends. The configuration tools developed in PRODNET, although designed for the creation phase, can be used here as well but some kind of expert assistance would be desirable at this phase. For instance, granting access to some information item or resource (during creation) can be done via a manual procedure because, in case of error, there will be feedback from the involved partner(s). However, for the cancellation phase it is more critical to give the company the confidence that everything the company wants to protect is in fact protected. Due to the time span between creation and dissolution, the human operators involved, even if the same, might not remember anymore all the granted rights. Therefore, a strong "memory link" between the two configuration phases is necessary.

There is also considerable knowledge that can be elicited from the ending cooperation experience, namely the knowledge about what went right, what went wrong, partners performance / reliability, jointly defined business process templates, etc. Defining the ownership and access rights to this knowledge is not an easy task and requires further investigation.

Only when these issues are better understood, it will be possible to specify which set of support services are necessary. Unfortunately this is an area almost unexplored by the recent research efforts. But understanding the mechanisms and requirements involved in the $\mathrm{VE}$ dissolution process and providing a set of computer-assisted tools is certainly a major step towards proper support for agility in virtual enterprises.

\section{CONCLUSIONS}

Support for agility is an important requirement in any infrastructure for virtual enterprises and its consequences are reflected at all phases of the VE life cycle.

A number of contributions in this direction were developed in the framework of two projects: PRODNET II and MASSYV, covering mostly the creation, operation, and reconfiguration phases of Virtual Enterprises. The paper addresses many of these developments.

Nevertheless more work on this subject is necessary, namely in terms of the high-level coordination, advanced cooperative information management, more flexible VE creation processes, and support for the VE dissolution phase.

Acknowledgements. The authors thank the European Commission for the partial funding for this work and their partners of the PRODNET II and MASSYVE projects for their valuable contributions.

\section{REFERENCES}

1. Afsarmanesh, H., Garita, C., Ugur, Y., Frenkel, A., and Hertzberger, L. O. - Design of the DIMS Architecture in PRODNET, in [5], 1999. 
2. Alonso, G. at al, - The WISE approach to Electronic Commerce, http://www.inf.ethz.ch/department/SS/iks/research/wise.html, Feb 15, 1999.

3. Alzaga, A.; Martin, J. - A design process model to support concurrent project development in networks of SMEs, in [5], 1999.

4. Bremer, C. F.; Mundim, A.; Michilini, F.; Siqueira, J.; Ortega, L. - A Brazilian case of VE coordination, in [5], 1999.

5. Camarinha-Matos, L. M., and Afsarmanesh, H. - Infrastructures for Virtual Enterprises - Networking Industrial Enterprises, Kluwer Academic Publishers, ISBN 0-7923-8639-6, Oct 1999.

6. Camarinha-Matos, L.M.; Cardoso, T. - Selection of partners for a virtual enterprise, in [5], 1999.

7. Camarinha-Matos, L. M.; Lima, C. - A framework for cooperation in virtual enterprises, Proceedings of DIISM'98 - Design of Information Infrastructure Systems for Manufacturing, Fort Worth, USA, May 1998.

8. Camarinha-Matos, L. M.; Lima, C. - PRODNET coordination module, in [5], 1999.

9. Damascos - http://www.damascos.com

10. Davis, R.; Smith, R. - Negotiation as a Metaphor for Distributed Problem Solving, Artificial Intelligence, 20, pp. 63-109, 1983.

11. Frenkel, A., Afsarmanesh, H., Garita, C., and Hertzberger, L. O. (2000) "Information Access Rights in Virtual Enterprises." Submitted to the 2nd IFIP / MASSYVE Working Conference on Infrastructures for Virtual Enterprises, Pro-VE 2000, Florianopolis, Brazil.

12. Garita, C., Afsarmanesh, H., and Hertzberger, L. O. (2000). "The PRODNET Federated Information Management Approach for Virtual Enterprise Support (to appear)." Journal of Intellingent Manufacturing.

13. Goranson, H.T. - The Agile Virtual Enterprise - Cases, metrics, tools. Quorum Books, 1999, ISBN 1-56720-264-0.

14. Kidd, P. - Agile Manufacturing: forging new frontiers, Addison-Wesley, 1994.

15. Klen, A. P.; Rabelo, R.J., Spinosa, L.M; Ferreira, A. C. - Integrated Logistics in the Virtual Enterprise: The PRODNET-II approach, Proceedings IMS'98 $-5^{\text {th }}$ IFAC Workshop on Intelligent Manufacturing Systems, pp. 289-295, Gramado - Brazil.

16. Klen, A. P.; Rabelo, R.J., Spinosa, L.M; Ferreira, A. C. (1999), Distributed Business Process Management, in [5], 1999.

17. Metes, G.; Gundry, J.; Bradish, P. - Agile Networking - Competing through the Internet and Intranets, Prentice Hall, 1998, ISBN 0-13-760125-5.

18. Molina, A.; Flores, M.; Caballero, D. - Virtual enterprises: A Mexican case study, in Intelligent Systems for Manufacturing, L.M. Camarinha-Matos et al. (Eds.), Kluwer Academic Publishers, ISBN 0-412-84670-5, 1998.

19. Rabelo, R.; Camarinha-Matos, L.M. - Towards Agile Scheduling in Extended Enterprise, em Balanced Automation Systems II - Implementation Challenges for Anthropocentric Manufacturing, Eds. Luis M. Camarinha-Matos e Hamideh Afsarmanesh, Chapman \& Hall, pp. 413-422, 1996.

20. Rabelo, R., Camarinha-Matos, L. M., and Afsarmanesh, H. - Multiagent-based Agile Scheduling, International Journal of Robotics and Autonomous Systems, Special Issue on Multi-Agent System Applications, 27(1-2), 15-28, 1998.

21. Rabelo, R. J.; Afsarmanesh, H., Camarinha-Matos, L.M. - Applying Federated Databases to Distributed Multi-agent Scheduling, Proceedings MAS'99 - 1st International IFAC Workshop on Multi-Agent Systems in Production, Vienna, Austria, pp. 277-282.

22. Rabelo, R. J.; Afsarmanesh, H., Camarinha-Matos, L.M. - Federated Multi-Agent Scheduling in Virtual Enterprises, submitted to PRO-VE'2000 2nd IFIP / MASSYVE Working Conference on Infrastructures for Virtual Enterprises - Managing Cooperation in Virtual Organizations and Electronic Business Towards Smart Organizations.

23. Smith, R. - The Contract Net Protocol: High-Level Communication and Control in a Distributed Problem Solver, IEEE Transactions on Systems, Man, and Cybernetics, 29, pp.1104-1113, 1980.

24. Spinosa, L.M.; Rabelo, R.J.; Klen, A. P. - High-Level Coordination of Business Processes in a Virtual Enterprise, in Globalization of Manufacturing in the Digital Communications Era of the 21st Century: Innovation, Agility, and the Virtual Enterprise, Edited by Jacucci, G., Olling, G.J., Preiss, K. and Wozny, M., Kluwer Academic Publishers, pp. 725-736, 1998.

25. SUN - JINI Technology Architectural Overview, Jan 1999, http://www.sun.com/jini/whitepapers/architecture.html.

26. WfMC - Workflow Management Coalition (1994) - The Workflow Reference Model - Document Nr. TC00 - 1003, Issue 1.1, Brussels Nov 29, 1994. 